\title{
Phylogenetic relationships of Malaysia's long-tailed macaques, Macaca fascicularis, based on cytochrome $b$ sequences
}

\author{
Muhammad Abu Bakar Abdul-Latiff', Farhani Ruslin', Vun Vui Fui',2, \\ Mohd-Hashim Abu', Jeffrine Japning Rovie-Ryan', Pazil Abdul-Patah ${ }^{1,3}$, \\ Maklarin Lakim ${ }^{4}$, Christian Roos ${ }^{5}$, Salmah Yaakop', Badrul Munir Md-Zain' \\ I School of Environmental and Natural Resource Sciences, Faculty of Science and Technology, Universiti Ke- \\ bangsaan Malaysia, 43600, Bangi, Selangor, Malaysia 2 UCSI University, 1, Jalan Menara Gading, UCSI \\ Heights, 56000, Cheras, Kuala Lumpur, Malaysia 3 Department of Wildlife and National Parks, Km 10, \\ Jalan Cheras, 50664, Kuala Lumpur, Malaysia 4 Sabah Parks, Research and Education Division, PO Box \\ 10626, 88806, Kota Kinabalu, Sabah, Malaysia 5 Gene Bank of Primates, German Primate Center, Leibniz \\ Institute for Primate Research, 37077 Göttingen, Germany \\ Corresponding author: Badrul Munir Md-Zain (abgbadd1966@yahoo.com) \\ Academic editor: M. Tocheri | Received 8 January 2014 | Accepted 22 April 2014 | Published 8 May 2014 \\ Citation: Abdul-Latiff MAB, Ruslin F, Vun VF, Mohd-Hashim A, Rovie-Ryan JJ, Abdul-Patah P, Lakim M, Roos C, \\ Yaakop S, Md-Zain BM (2014) Phylogenetic relationships of Malaysia's long-tailed macaques, Macaca fascicularis, based \\ on cytochrome $b$ sequences. ZooKeys 407: 121-140. doi: 10.3897/zookeys.407.6982
}

\begin{abstract}
Phylogenetic relationships among Malaysia's long-tailed macaques have yet to be established, despite abundant genetic studies of the species worldwide. The aims of this study are to examine the phylogenetic relationships of Macaca fascicularis in Malaysia and to test its classification as a morphological subspecies. A total of 25 genetic samples of $M$. fascicularis yielding $383 \mathrm{bp}$ of Cytochrome $b$ (Cyt $b$ ) sequences were used in phylogenetic analysis along with one sample each of $M$. nemestrina and $M$. arctoides used as outgroups. Sequence character analysis reveals that Cyt $b$ locus is a highly conserved region with only $23 \%$ parsimony informative character detected among ingroups. Further analysis indicates a clear separation between populations originating from different regions; the Malay Peninsula versus Borneo Insular, the East Coast versus West Coast of the Malay Peninsula, and the island versus mainland Malay Peninsula populations. Phylogenetic trees (NJ, MP and Bayesian) portray a consistent clustering paradigm as Borneo's population was distinguished from Peninsula's population ( $99 \%$ and $100 \%$ bootstrap value in NJ and MP respectively and 1.00 posterior probability in Bayesian trees). The East coast population was separated from other Peninsula populations ( $64 \%$ in NJ, 66\% in MP and 0.53 posterior probability in Bayesian). West coast populations were divided into 2 clades: the North-South (47\%/54\% in NJ, 26/26\%
\end{abstract}

Copyright M.A.B.Abdul-Latiff et al. This is an open access article distributed under the terms of the Creative Commons Attribution License (CC BY 4.0), which permits unrestricted use, distribution, and reproduction in any medium, provided the original author and source are credited. 
in MP and 1.00/0.80 posterior probability in Bayesian) and Island-Mainland (93\% in NJ, $90 \%$ in MP and 1.00 posterior probability in Bayesian). The results confirm the previous morphological assignment of 2 subspecies, $M$. f. fascicularis and $M$. f. argentimembris, in the Malay Peninsula. These populations should be treated as separate genetic entities in order to conserve the genetic diversity of Malaysia's $M$. fascicularis. These findings are crucial in aiding the conservation management and translocation process of $M$. fascicularis populations in Malaysia.

\section{Keywords}

Long-tailed macaque, Macaca fascicularis, Cytochrome $b$, phylogenetic relationships

\section{Introduction}

Macaca fascicularis (Raffles, 1821) is also known as long-tailed, crab-eating or cynomolgus macaque. This species is well distributed in the countries of Malaysia, Brunei, Bangladesh, Cambodia, Nicobar Islands, Indonesia, Lao PDR, Myanmar, Philippines, Singapore, Thailand, Timor-Leste and Vietnam (Figure 1) (Gumert et al. 2011). There appears to be a hybrid zone between $M$. fascicularis and M. mulatta (Zimmermann, 1780) in the northern range above mainland Southeast Asia, which makes it difficult to determine the northern distribution limit of M. fascicularis (Fooden, 1996). The distribution of long-tailed macaques was extended to the Pacific Ocean (Palau) (Crombie and Pregill 1999), Indian Ocean (Mauritius) (Trask et al. 2013) and New Guinea (Kemp and Burnett 2003) due to human-mediated introduction of the species to these respective regions in the recent past.

At least 10 subspecies of $M$. fascicularis are presently recognized; $M$. $f$. atriceps (Kloss, 1919), M. f. aurea (Geoffroy, 1831), M. f. condorensis (Kloss, 1926), M. f. fascicularis (Raffles, 1821), M.f. fusca (Miller, 1903), M.f. karimondjawae (Sody, 1949), M. f. lasiae (Lyon, 1916), M. f. philippinensis (Geoffroy, 1843), M. f. tua (Kellog, 1944) and M.f. umbrosa (Miller, 1902) (Groves 2001; Brandon-Jones et al. 2004) based on their morphological characteristics. These subspecies classifications were distinguished based on three critical aspects: tail length, pelage coloration and form of the cheek whiskers (Groves 2001). Both Groves (2001) and Brandon-Jones et al. (2004) agreed that only one subspecies, $M$. f. fascicularis, is distributed in Peninsula Malaysia and Borneo. Medway (1969), on the other hand, has acknowledged 3 subspecies of $M$. fascicularis distributed in Malaysia, specifically, M. f. fascicularis (Raffles, 1821) (Peninsula Malaysia); $M . f$. argentimembris (Kloss, 1911) (Redang Island) and M. $f$. laeta (Elliot, 1909) (Tioman Island and Tinggi Island). Raven (1935) acknowledged M. f. argentimembris by Kloss, 1911 observed in Redang Island as subspecies distributed in East Coast of Peninsula Malaysia. Weitzel et al. (1988) also acknowledged the distribution of M. f. laeta by Elliot (1909) observed in Tioman Island and Tinggi Island as subspecies distributed in the East Coast of Peninsula Malaysia.

Zhang et al. (1993) conducted one of the earliest thorough studies on the phylogeny of $M$. fascicularis that exploited mitochondrial DNA (mtDNA) using restriction endonuclease analysis. Smith et al. (2007) studied mtDNA variation within and among 


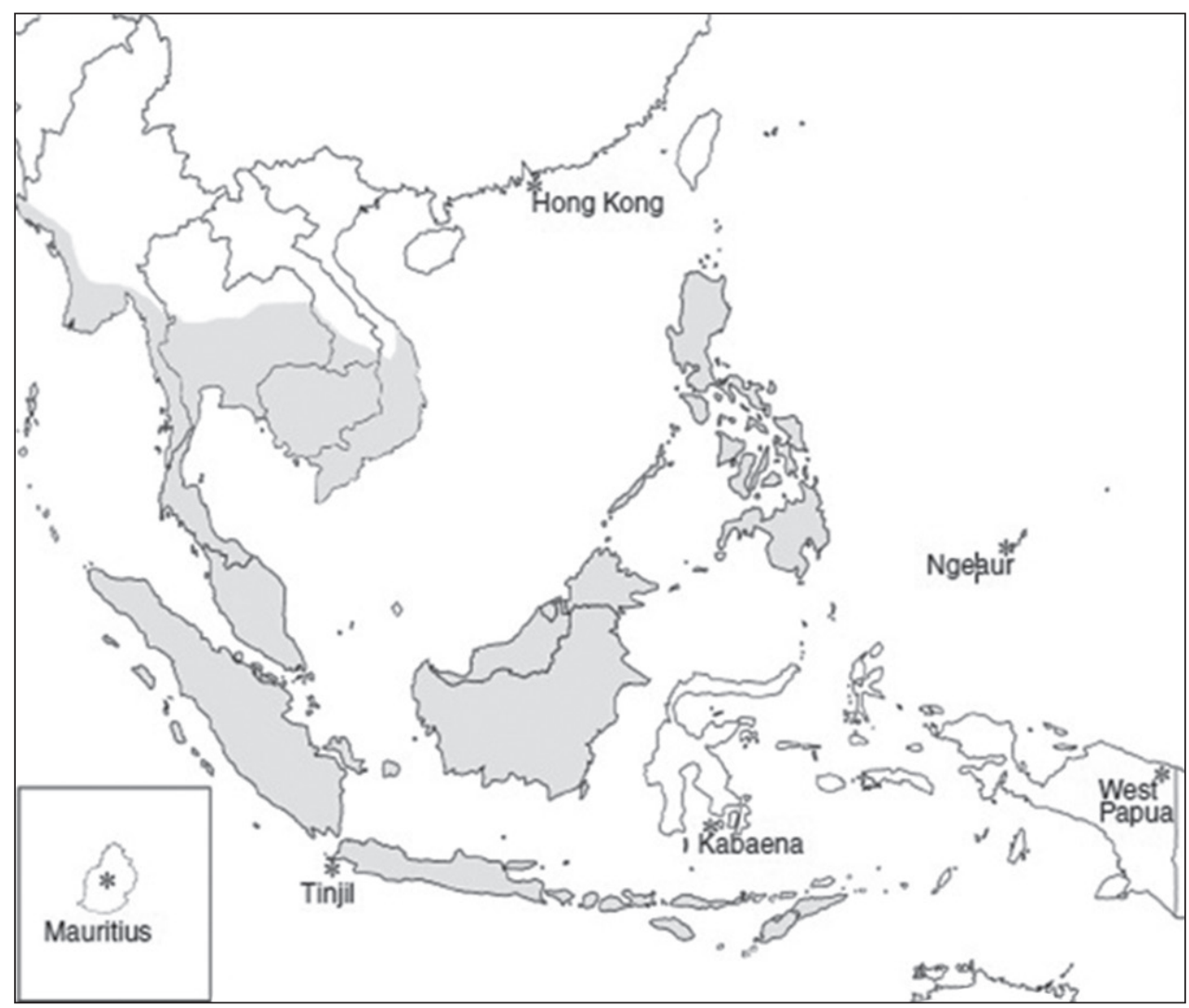

Figure I. Distribution of the long-tailed macaque (M. fascicularis) in Southeast Asia (Gumert et al. 2011).

regional populations of $M$. fascicularis by applying an astonishing 1053 samples comprising 5 regional populations (Malaysia, Indonesia, Indochina, the Philippines and Mauritius). Deinard and Smith (2001) screened the nuclear DNA sequences (natural resistance-associated macrophage protein 1, NRAMP1) of 59 individuals representing 11 species of macaques, and their results suggest that $M$. fascicularis may not be as primitive as the mtDNA data suggests. Numerous other genetic studies on $M$. fascicularis have been conducted. Tosi et al. (2002) determined the introgression between M. fascicularis and M. mulatta using Y-chromosome and mitochondrial markers. Otting et al. (2009) studied the haplotypes in pedigreed cynomolgus macaques. Street et al. (2007) analyzed the nucleotide polymorphisms in M. mulatta and M. fascicularis. Blancher et al. (2008) described the phylogeny of 4 populations of $M$. f. fascicularis (Indonesia, Indochina, Philippines and Mauritius). Stevison and Kohn (2009) conducted genetic analysis to determine hybridization between rhesus and long-tailed macaques. Finally, Md-Zain et al. (2010a) determined the phylogenetic relationships of Cercopithecidae using $M$. fascicularis as a representative.

Despite the abundance of genetic studies on or including $M$. fascicularis, the phylogenetic relationships among Malaysia's long-tailed macaques remain uncertain. 
However, these phylogenetic data are crucial for conservation management of $M$. fascicularis as this species is reported as a pest in human settlement areas (Md-Zain et al. 2010b; 2011). For example, M. fascicularis engages frequently in crop-raiding activities, and these behaviors are often reinforced by humans that feed these macaques either directly or indirectly, which leads to unintentional habituation of the species. The annual report by the Department of Wildlife and National Parks (PERHILITAN) (2010) indicated that $M$. fascicularis is at the top of the human-wildlife conflict species case list. From a recorded 9,286 complaints of wildlife disturbance from various species, complaints on $M$. fascicularis disturbance were the highest, with 5,930 complaints (63.86\%). The phylogenetic relationships of Malaysia's $M$. fascicularis data are crucial in planning and executing the translocation process of this species in the future, which is one of the major actions in the conservation management and human-wildlife conflict management of the species. By understanding the phylogenetic relationships of Malaysia's crab-eating macaque, the plan for translocation the species can finally be carried out without a risk of losing important genetic diversity or even unique genetic lineages of the species.

Phylogenetic studies at the subspecies level are very scarce (Rosli et al. 2014), as many primatologists are making the species group classifications the focal point in their studies. Both Vun et al. (2011) and Blancher et al. (2008) have successfully explained the phylogenetic relationships of Presbytis and $M$. fascicularis, respectively, at population level using Cyt $b$ sequences, proving that Cyt $b$ is a suitable locus for population level studies of Cercopithecidae. Thus, the objectives of this research are to determine the phylogenetic relationships of Malaysia's $M$. fascicularis using mitochondrial Cyt $b$ sequences and to test genetically the subspecies classifications as made by Medway (1969), Groves (2001) and Brandon-Jones et al. (2004) based on morphological characteristics.

\section{Methods}

\section{DNA extraction, polymerase chain reaction (PCR) and sequencing}

Altogether, 27 genetic samples (Table 1 and Figure 2) were used in this research. These samples were provided by the Department of Wildlife and National Parks (PERHILITAN) and Sabah Parks. The samples derive mainly from feces collected in the original habitats of $M$. fascicularis. In addition, blood and tissue samples collected from a roadkill specimen of $M$. fascicularis were also used in this study. Mitochondrial DNA (mtDNA) was extracted from each genetic sample using QIAGEN DNeasy Blood and Tissue Kit, following the manufacturer's protocol. A mtDNA genome from FTA (fast technology for analysis of nucleic acids) sample was extracted using the WHATMAN ${ }^{\circ}$ GenSolve Recovery Kit, also following the manufacturer's protocol. DNA was extracted from $0.5 \mathrm{~g}-1.0 \mathrm{~g}$ of fecal sample using innuPREP Stool DNA kit (Analytik Jena) following the manufacturer's protocol. 
Table I. Details on the samples used in this study

\begin{tabular}{|c|c|c|c|}
\hline No. & Sample name & Taxon & Locality \\
\hline 1 & MF135 & Macaca fascicularis fascicularis & Port Dickson, Negeri Sembilan \\
\hline 2 & MF136 & Macaca fascicularis fascicularis & Port Dickson, Negeri Sembilan \\
\hline 3 & MF137 & Macaca fascicularis fascicularis & Port Dickson, Negeri Sembilan \\
\hline 4 & MF138 & Macaca fascicularis fascicularis & Port Dickson, Negeri Sembilan \\
\hline 5 & M1 & Macaca fascicularis fascicularis & Bangi, Selangor \\
\hline 6 & BM95 & Macaca fascicularis fascicularis & Kluang, Johor \\
\hline 7 & MF488 & Macaca fascicularis fascicularis & Tanjung Tokong. Pulau Pinang \\
\hline 8 & MF489 & Macaca fascicularis fascicularis & Tanjung Tokong. Pulau Pinang \\
\hline 9 & MF490 & Macaca fascicularis fascicularis & Tanjung Tokong. Pulau Pinang \\
\hline 10 & MF491 & Macaca fascicularis fascicularis & Tanjung Tokong. Pulau Pinang \\
\hline 11 & MF719 & Macaca fascicularis fascicularis & Pantai Remis, Perak \\
\hline 12 & MF720 & Macaca fascicularis fascicularis & Pantai Remis, Perak \\
\hline 13 & MF721 & Macaca fascicularis fascicularis & Pantai Remis, Perak \\
\hline 14 & MF722 & Macaca fascicularis fascicularis & Pantai Remis, Perak \\
\hline 15 & ALMFD16 & Macaca fascicularis fascicularis & Pasir Mas, Kelantan \\
\hline 16 & ALMFD17 & Macaca fascicularis fascicularis & Pasir Mas, Kelantan \\
\hline 17 & ALMFD28 & Macaca fascicularis fascicularis & Pasir Mas, Kelantan \\
\hline 18 & ALMFD29 & Macaca fascicularis fascicularis & Pasir Mas, Kelantan \\
\hline 19 & ALMFA62 & Macaca fascicularis fascicularis & Kuala Gula, Perak \\
\hline 20 & ALMFA63 & Macaca fascicularis fascicularis & Kuala Gula, Perak \\
\hline 21 & ALMFA64 & Macaca fascicularis fascicularis & Kuala Gula, Perak \\
\hline 22 & ALMFA65 & Macaca fascicularis fascicularis & Kuala Gula, Perak \\
\hline 23 & MF03 & Macaca fascicularis fascicularis & Pulau Sapi, Sabah \\
\hline 24 & MF04 & Macaca fascicularis fascicularis & Pulau Sapi, Sabah \\
\hline 25 & MF05 & Macaca fascicularis fascicularis & Pulau Sapi, Sabah \\
\hline 26 & BM97 & Macaca nemestrina & Zoo Taiping, Perak \\
\hline 27 & BM104 & Macaca arctoides & Malacca Zoo \\
\hline
\end{tabular}

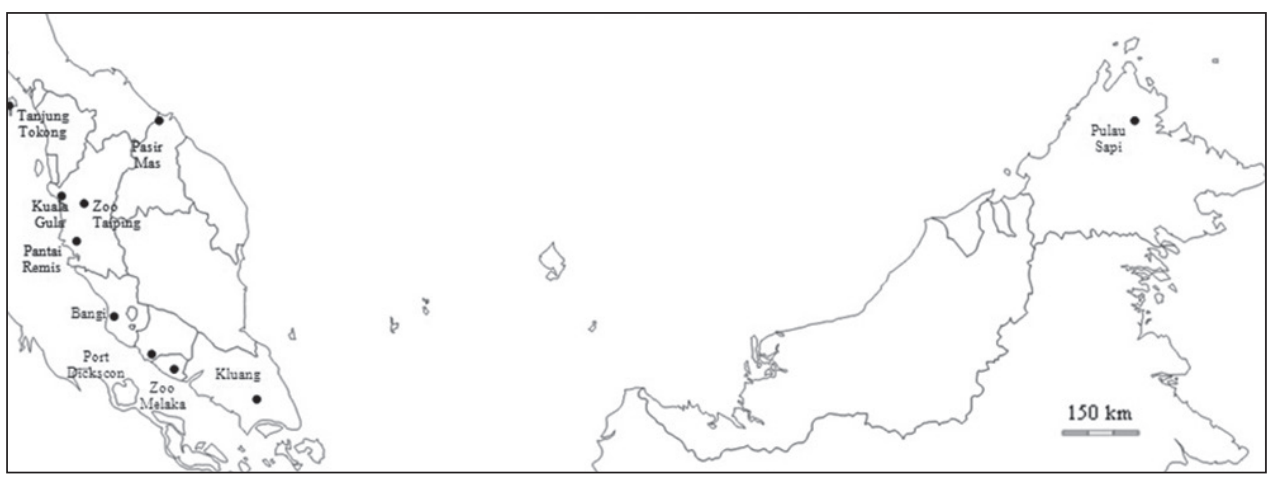

Figure 2. Sampling location of M. fascicularis throughout Peninsula Malaysia and Borneo.

Polymerase Chain Reaction (PCR) was employed in order to amplify the targeted locus in the mtDNA genome, which is a partial fragment of the Cyt $b$ gene, by using Mastercycler ${ }^{\circledast}$ nexus (Eppendorf North America, Inc.). PCR was performed by using 
Phusion $^{\text {TM }}$ Flash High-Fidelity PCR Master Mix (Finnzymes, OY), which has extreme speed (extension times of $15 \mathrm{~s} / \mathrm{kb}$ or less), high accuracy (proofreads DNA polymerase with a fidelity of $25 \mathrm{X} \mathrm{Taq}$ polymerase) and a very high yield in reduced times. Primers used in this study were (L14724) 5'- CGAAGCTTGATATGAAAAACCATCGTTG -3' (Pääbo and Wilson 1988) and (H15149) 5'-AAACTGCAGCCCCTCAGAATGATATTTGTCCTCA - 3' (Kocher et al. 1989). PCR reactions were carried out under the following parameters: $98^{\circ} \mathrm{C}$ initial denaturation for 30 seconds, followed by 30 cycles of $98^{\circ} \mathrm{C}$ denaturation for 10 seconds, $55^{\circ} \mathrm{C}$ of annealing for 30 seconds, $72^{\circ} \mathrm{C}$ extension for 30 seconds and the final extension stage at $72^{\circ} \mathrm{C}$ for 10 minutes. The PCR product was purified using the Vivantis G-F1 PCR Clean-up Kit, and the purified PCR products were sent to the $1^{\text {st }}$ Base Laboratories Sdn Bhd (Malaysia) for sequencing.

\section{Sequence and phylogenetic analysis}

Sequence results obtained from the $1^{\text {st }}$ Base Laboratories Sdn Bhd were proofread and edited using Bioedit Sequence Alignment Editor, and the Sequence Similarity searches were performed using GenBank BLASTn application to validate the DNA sequences obtained. DNA sequences were submitted to GenBank under accession number KJ592589-KJ592594. Bioedit's ClustalW multiple alignment algorithm was then used to align the sequence results, and sequence analysis and phylogenetic analysis were performed. DNASP 4.0 (Rozas et al. 2003), PAUP 4.0b10 (Swofford 2002) and MEGA version 4.0 (Tamura et al. 2007) software were used for sequence analysis to determine nucleotide diversity $(\pi)$ and net nucleotide divergence $(\mathrm{Da})$; genetic distance and single nucleotide polymorphisms (SNPs) of the sequences/datasets respectively.

Three methods of phylogenetic tree reconstructions were carried out; the distancebased method (neighbor joining, NJ) using MEGA version 4.0 (Tamura et al. 2007); the character-based method (maximum parsimony, MP) using Phylogenetic Analysis Using Parsimony (PAUP) version 4.0b10 (Swofford 2002) and Bayesian inference using MrBayes 3.1 (Huelsenbeck and Ronquist 2001). The Kimura-2-Parameter model was selected for NJ phylogenetic reconstructions. MP phylogenetic tree was carried out with heuristic search methods and 1000 random stepwise addition with the application of a $50 \%$ consensus-majority rule concept (Swofford 2002). In the MP analysis, each transition and transversion was calculated on average. The MP phylogenetic tree was constructed using a tree bisection and reconnection (TBR) algorithm, and all the trees constructed underwent 1000 bootstrap replications to obtain the bootstrap confidence level.

Modeltest version 3.7 software (Posada and Crandall 1998) was used to select the best substitution model for the partial Cyt $b$ sequences using Akaike Information Criterion (AIC). The best substitution model was applied in the Bayesian analysis using MrBayes 3.1.2. software. The most suitable model that fit the data was the HKY+G model with a gamma shape parameter of 0.5455 and base frequencies of 0.2866 for A, 0.3171 for C, 0.1266 for $\mathrm{G}$ and 0.2696 for T. We ran Metropolis-coupled Markov Chain Monte Carlo (MCMC) with 300000 generations, with 0.008214 Split Fre- 
quencies Probability $(\mathrm{P})$ and tree was sampled every 10 generations. The first $25 \%$ of the trees obtained in the analysis was discarded as burn-in (7500 trees discarded from total 30000 trees), a majority-rule consensus of remaining trees was constructed and posterior probabilities (PP) were summarized for each branch.

\section{Results}

Comparison of the cytochrome $b$ sequence of Malaysia's $M$. fascicularis with GenBank's sequence

Partial sequences of Cyt $b$ locus in size of 383 bp were successfully sequenced for all 27 genetic samples (Table 1). The first analysis conducted on the sequences consisted of sequence similarity searches using the GenBank application to validate each sequence obtained was from the correct taxon of the acquired samples and to avoid encountering the data problem of nuclear insertion. All genetic samples matched the target species sequences in GenBank with samples FJ906803.1 (M. fascicularis complete genome) and corresponded to most of the ingroup samples with average query cover and maximum identities scores at $95 \%$ and $97 \%$, respectively.

\section{Sequence polymorphism, genetic distance and nucleotide diversity}

A total of 27 genetic sequences in a size of 383 bp of Cyt $b$ locus yielded 78 (20.37\%) variable sites, of which 34 sites were parsimony informative characters (8.88\%). Interestingly, when outgroup samples ( $M$. nemestrina and $M$. arctoides) were excluded from the analysis, only $23(6 \%)$ variable sites were detected, all of which were parsimony informative characters. From these 23 informative characters, 13 were generated by the inclusion of Borneo samples in the analysis; whereas, if the Borneo samples were excluded, only $10(2.6 \%)$ parsimony informative characters were detected.

Pairwise genetic distances of Cyt $b$ partial sequences were calculated with PAUP $4.0 \mathrm{~b} 10$ (Swofford 2002) using the Kimura-2-Parameter model (Table 2). The genetic distance of samples originating from Borneo and Peninsula Malaysia showed a minimum genetic distance of 0.04068 (BM95) and a maximum value as high as 0.049 (ALMFA62, ALMFA63, ALMFA64 and ALMFA65), which is the highest value within the M. fascicularis genetic distance analysis. The genetic distance of samples originating from the East Coast of Peninsula Malaysia and West Coast of Peninsula Malaysia (excluding samples from Borneo) showed the minimum value of genetic distance was 0.008 (BM95), and the maximum value was 0.016 (ALMFA62, ALMFA63, ALMFA64 and ALMFA65). The separation of the island population of $M$. fascicularis and the mainland of Peninsula Malaysia populations (excluding samples from Borneo) showed a minimum value of genetic distance of 0.008 (MF719, MF720, MF721 and MF722) and a maximum value of genetic distance of 0.016 (MF135, MF136, MF137 and MF138 and M1). 


\begin{tabular}{|c|c|c|c|c|c|c|c|c|c|c|c|c|c|c|c|c|c|c|c|c|c|c|c|c|c|c|}
\hline$\searrow$ & & & & & & & & & & & & & & & $\begin{array}{l}8 \\
\vdots \\
\vdots \\
\vdots \\
\vdots \\
0\end{array}$ & $\left|\begin{array}{|c}0 \\
\vdots \\
0 \\
0 \\
0\end{array}\right|$ & $\begin{array}{l}8 \\
\vdots \\
\vdots \\
\vdots \\
0\end{array}$ & $\begin{array}{l}\tilde{a} \\
\tilde{n} \\
\vdots \\
\vdots \\
0\end{array}$ & $\begin{array}{l}\tilde{z} \\
\tilde{\sigma} \\
\vdots \\
0\end{array}$ & $\begin{array}{l}\tilde{\imath} \\
\tilde{a} \\
\vdots \\
0 \\
0\end{array}$ & $\begin{array}{l}\tilde{\imath} \\
\tilde{z} \\
\vdots \\
\dot{0}\end{array}$ & & 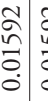 & 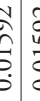 & & $\begin{array}{l}\infty \\
b \\
b \\
b\end{array}$ \\
\hline 2 & & & & & & & & & & & & & & 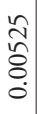 & 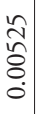 & $\begin{array}{l}\varkappa \\
\hat{\tilde{z}} \\
\delta \\
0\end{array}$ & $\begin{array}{l}\tilde{\approx} \\
\hat{\tilde{o}} \\
0 \\
0\end{array}$ & 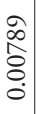 & $\begin{array}{l}0 \\
0 \\
\hat{\Xi} \\
0 \\
0\end{array}$ & $\begin{array}{l}\grave{o} \\
\hat{o} \\
0 \\
\dot{0}\end{array}$ & 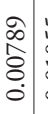 & & $\begin{array}{l}n \\
\tilde{c} \\
\vdots \\
0 \\
0\end{array}$ & $\begin{array}{l}\hat{b} \\
\vdots \\
0 \\
0 \\
.\end{array}$ & & 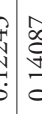 \\
\hline 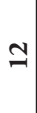 & & & & & & & & & & & & & $\begin{array}{l}\text { ¿े } \\
\vdots \\
\circ\end{array}$ & $\begin{array}{l}\text { ते } \\
\text { है } \\
0 \\
0\end{array}$ & $\begin{array}{l}\tilde{n} \\
\tilde{\tilde{o}} \\
\vdots \\
0 \\
0\end{array}$ & $\begin{array}{l}\tilde{\hat{n}} \\
\hat{\delta} \\
0 \\
0\end{array}$ & $\begin{array}{l}\tilde{n} \\
\tilde{z} \\
\vdots \\
0\end{array}$ & 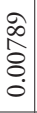 & 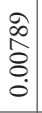 & $\begin{array}{l}2 \\
\infty \\
\delta \\
0 \\
\dot{0}\end{array}$ & 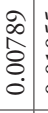 & $\begin{array}{l}n \\
\tilde{c} \\
0 \\
0 \\
0\end{array}$ & $\begin{array}{l}\tilde{n} \\
\hat{\sigma} \\
\vdots \\
0 \\
0\end{array}$ & $\begin{array}{l}\hat{n} \\
\vdots \\
0 \\
0\end{array}$ & $\begin{array}{l}\hat{b} \\
\vdots \\
\vdots \\
0 \\
\vdots\end{array}$ & 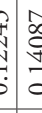 \\
\hline$z$ & & & & & & & & & & & & $\begin{array}{l}8 \\
\vdots \\
\vdots \\
\vdots\end{array}$ & $\begin{array}{l}\text { ¿े } \\
\vdots \\
0\end{array}$ & 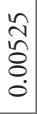 & $\begin{array}{l}\tilde{w} \\
\tilde{z} \\
\vdots \\
0 \\
0\end{array}$ & $\begin{array}{l}\tilde{\hat{z}} \\
\hat{\delta} \\
\dot{0}\end{array}$ & $\begin{array}{l}\tilde{y} \\
\hat{\imath} \\
\vdots \\
0 \\
0\end{array}$ & 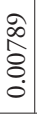 & $\begin{array}{l}\infty \\
\infty \\
\stackrel{0}{0} \\
\dot{0}\end{array}$ & $\begin{array}{l}a \\
0 \\
\hat{o} \\
0\end{array}$ & $\begin{array}{l}\grave{0} \\
\vdots \\
\vdots \\
0\end{array}$ & $\begin{array}{l}\tilde{n} \\
\hat{\sigma} \\
\vdots \\
\dot{0}\end{array}$ & $\begin{array}{l}\tilde{\hat{c}} \\
\hat{n} \\
\dot{0}\end{array}$ & 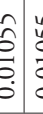 & $\begin{array}{ll}\hat{c} \\
\vdots \\
\vdots \\
0\end{array}$ & 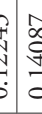 \\
\hline$\stackrel{ }{1}$ & & & & & & & & & & & & $\begin{array}{l}\stackrel{8}{0} \\
\stackrel{0}{0}\end{array}$ & $\begin{array}{l}\stackrel{8}{0} \\
\vdots \\
\vdots\end{array}$ & 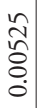 & $\begin{array}{l}\tilde{\hat{w}} \\
\tilde{\delta} \\
\vdots \\
\dot{0}\end{array}$ & $\begin{array}{l}\tilde{\hat{~}} \\
\tilde{\delta} \\
0 \\
0\end{array}$ & $\begin{array}{l}\tilde{w} \\
\tilde{o} \\
\vdots \\
0\end{array}$ & $\begin{array}{l}\grave{\alpha} \\
\hat{\sigma} \\
\dot{0} \\
\dot{0}\end{array}$ & 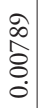 & $\begin{array}{l}\grave{D} \\
\hat{b} \\
\vdots \\
\dot{0}\end{array}$ & $\begin{array}{c}\dot{0} \\
\hat{o} \\
\vdots \\
\dot{0}\end{array}$ & $\begin{array}{l}\tilde{n} \\
\hat{o} \\
\vdots \\
0 \\
0\end{array}$ & $\begin{array}{l}\tilde{n} \\
\tilde{c} \\
\vdots \\
0 \\
0\end{array}$ & $\begin{array}{c}\hat{c} \\
b \\
\vdots \\
0\end{array}$ & 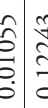 & 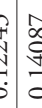 \\
\hline$a$ & & & & & & & & & & & 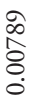 & 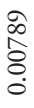 & $\begin{array}{l}\stackrel{\alpha}{\infty} \\
\hat{0} \\
0 \\
0\end{array}$ & 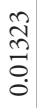 & $\begin{array}{l}\tilde{z} \\
\tilde{\Xi} \\
\vdots \\
0 \\
0\end{array}$ & $\begin{array}{l}\approx \\
\tilde{a} \\
0 \\
0 \\
0\end{array}$ & $\begin{array}{l}\approx \\
\tilde{a} \\
\tilde{0} \\
0\end{array}$ & $\begin{array}{l}\tilde{\sigma} \\
\tilde{n} \\
\vdots \\
0 \\
0\end{array}$ & 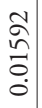 & $\begin{array}{l}\tilde{n} \\
\tilde{\sigma} \\
0\end{array}$ & $\begin{array}{l}\tilde{\sigma} \\
\tilde{n} \\
\tilde{o} \\
\dot{0}\end{array}$ & $\begin{array}{l}\tilde{2} \\
\tilde{\Xi} \\
\vdots \\
\dot{0}\end{array}$ & 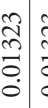 & 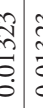 & $\begin{array}{c}2 \\
c \\
c \\
0 \\
0\end{array}$ & 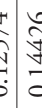 \\
\hline$\infty$ & & & & & & & & & & $\begin{array}{l}0 \\
0 \\
0\end{array}$ & $\begin{array}{l}\stackrel{\infty}{\circ} \\
\stackrel{8}{2}\end{array}$ & 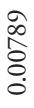 & $\begin{array}{l}\stackrel{\infty}{\infty} \\
\hat{\delta} \\
0 \\
0\end{array}$ & 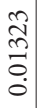 & $\begin{array}{l}\tilde{z} \\
\tilde{z} \\
\vdots \\
0 \\
0\end{array}$ & $\begin{array}{l}\approx \\
\tilde{a} \\
0 \\
0 \\
0\end{array}$ & $\begin{array}{l}\approx \\
\tilde{n} \\
\tilde{\Xi} \\
0\end{array}$ & $\begin{array}{l}\tilde{\sigma} \\
\tilde{n} \\
\vdots \\
0 \\
0\end{array}$ & $\begin{array}{l}\tilde{\sigma} \\
\tilde{ٍ} \\
\stackrel{0}{0}\end{array}$ & $\begin{array}{l}\tilde{n} \\
\tilde{a} \\
\dot{0}\end{array}$ & $\begin{array}{l}\tilde{\sigma} \\
\tilde{n} \\
\tilde{a} \\
\dot{0}\end{array}$ & $\begin{array}{l}\tilde{2} \\
\tilde{n} \\
\vdots \\
\dot{0}\end{array}$ & 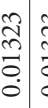 & $\begin{array}{c}m \\
- \\
c \\
\dot{0}\end{array}$ & $\begin{array}{c}c \\
c \\
c \\
c \\
0\end{array}$ & $\begin{array}{l}+1 \\
\vdots \\
\vdots\end{array}$ \\
\hline$\Lambda$ & & & & & & & & 8 & & 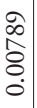 & $\begin{array}{l}\text { के } \\
\hat{8}\end{array}$ & $\begin{array}{l}\stackrel{o}{\infty} \\
\hat{o} \\
\stackrel{0}{0}\end{array}$ & $\begin{array}{l}\stackrel{0}{0} \\
\hat{8} \\
\dot{0}\end{array}$ & 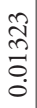 & $\begin{array}{l}\tilde{a} \\
\widetilde{a} \\
\vdots \\
0 \\
0\end{array}$ & $\begin{array}{l}\approx \\
\tilde{0} \\
0 \\
0\end{array}$ & $\begin{array}{l}\approx \\
\tilde{n} \\
\tilde{0} \\
\dot{0}\end{array}$ & $\begin{array}{l}\tilde{\sigma} \\
\tilde{\widehat{\sigma}} \\
0 \\
0\end{array}$ & 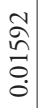 & $\begin{array}{l}\tilde{\omega} \\
\tilde{n} \\
0 \\
0\end{array}$ & $\begin{array}{l}\tilde{\Omega} \\
\tilde{n} \\
\tilde{o} \\
\dot{0}\end{array}$ & $\begin{array}{l}\tilde{z} \\
\tilde{c} \\
\tilde{0} \\
\dot{0}\end{array}$ & 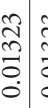 & $\begin{array}{c}\tilde{c} \\
\tilde{0} \\
\stackrel{0}{0}\end{array}$ & $\begin{array}{c}c \\
c \\
c \\
0 \\
0\end{array}$ & 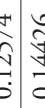 \\
\hline ( & & & & & & & & 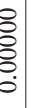 & छ & $\begin{array}{l}a \\
\text { ô } \\
0\end{array}$ & $\begin{array}{l}\text { के } \\
\hat{8}\end{array}$ & 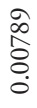 & $\begin{array}{l}\infty \\
\infty \\
\stackrel{0}{0} \\
0\end{array}$ & 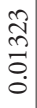 & $\begin{array}{l}\tilde{z} \\
\tilde{\Xi} \\
\vdots \\
0 \\
0\end{array}$ & $\begin{array}{l}\tilde{a} \\
\tilde{0} \\
0 \\
0\end{array}$ & $\begin{array}{l}\widetilde{N} \\
\tilde{n} \\
\vdots \\
0\end{array}$ & 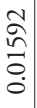 & $\begin{array}{c}\tilde{\sigma} \\
\hat{n} \\
\vdots \\
0\end{array}$ & $\begin{array}{l}\tilde{\sigma} \\
\vdots \\
0 \\
0\end{array}$ & $\begin{array}{l}\tilde{\sigma} \\
\tilde{n} \\
\vdots \\
0 \\
0\end{array}$ & $\begin{array}{l}2 \\
\tilde{z} \\
\tilde{\vdots} \\
\dot{0} \\
0\end{array}$ & $\begin{array}{c}\tilde{2} \\
\tilde{z} \\
\vdots \\
0 \\
0\end{array}$ & 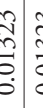 & 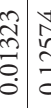 & 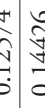 \\
\hline$n$ & & & & & & $\begin{array}{l}8 \\
\vdots \\
\vdots \\
\vdots \\
\end{array}$ & 8 & $\frac{8}{8}$ & छ & 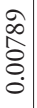 & $\begin{array}{l}\stackrel{\infty}{ } \\
\frac{8}{2}\end{array}$ & 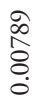 & 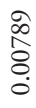 & $\begin{array}{l}\approx \\
\tilde{\omega} \\
\vdots \\
0 \\
0\end{array}$ & $\begin{array}{l}\tilde{z} \\
\tilde{z} \\
\vdots \\
\dot{0}\end{array}$ & $\begin{array}{l}\approx \\
\tilde{\Xi} \\
0 \\
0\end{array}$ & $\begin{array}{l}\approx \\
\tilde{n} \\
\vdots \\
0\end{array}$ & $\begin{array}{l}\tilde{\sigma} \\
\tilde{\widehat{n}} \\
0 \\
0\end{array}$ & $\begin{array}{l}\tilde{\Omega} \\
\stackrel{\Omega}{0} \\
0\end{array}$ & $\begin{array}{l}\tilde{\hat{n}} \\
\tilde{0} \\
0\end{array}$ & $\begin{array}{l}\tilde{\sigma} \\
\tilde{n} \\
\tilde{o} \\
\dot{0}\end{array}$ & $\begin{array}{l}\tilde{Z} \\
\tilde{z} \\
\vdots \\
0 \\
\dot{0}\end{array}$ & $\begin{array}{l}\tilde{a} \\
\tilde{n} \\
\vdots \\
0 \\
0\end{array}$ & 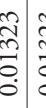 & $\begin{array}{c}2 \\
c \\
c \\
0 \\
0 \\
0\end{array}$ & 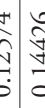 \\
\hline 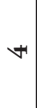 & & & & 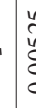 & 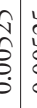 & $\begin{array}{l}\tilde{\hat{n}} \\
\hat{z} \\
0 \\
0\end{array}$ & $\begin{array}{l}\hat{\delta} \\
\hat{\delta}\end{array}$ & है & $\approx$ & 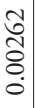 & $\begin{array}{l}\text { तु } \\
\text { రิ }\end{array}$ & 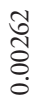 & 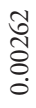 & $\begin{array}{l}\stackrel{\partial}{ } \\
\hat{\Xi} \\
\dot{0}\end{array}$ & $\begin{array}{l}\stackrel{a}{\alpha} \\
\hat{\Xi} \\
\dot{0}\end{array}$ & $\begin{array}{l}2 \\
\infty \\
\hat{0} \\
0 \\
0\end{array}$ & $\begin{array}{l}\stackrel{\alpha}{\infty} \\
\hat{0} \\
0 \\
0\end{array}$ & $\begin{array}{l}\tilde{n} \\
\tilde{o} \\
0 \\
0 \\
0\end{array}$ & $\begin{array}{l}\tilde{n} \\
\hat{o} \\
0 \\
0 \\
0\end{array}$ & $\begin{array}{l}\tilde{n} \\
\hat{0} \\
0 \\
0\end{array}$ & $\begin{array}{l}\tilde{n} \\
\hat{o} \\
0 \\
0 \\
0\end{array}$ & 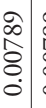 & 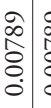 & 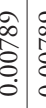 & 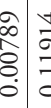 & 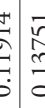 \\
\hline m & & & 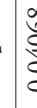 & 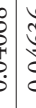 & $\begin{array}{l}0 \\
0 \\
\vdots \\
0 \\
0 \\
\vdots\end{array}$ & \begin{tabular}{l}
0 \\
\multirow{6}{0}{} \\
$\vdots$ \\
0 \\
0
\end{tabular} & 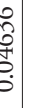 & 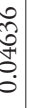 & 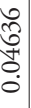 & 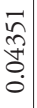 & \begin{tabular}{l}
$\overrightarrow{\widetilde{\sigma}}$ \\
\multirow{\sigma}{*}{}
\end{tabular} & 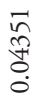 & 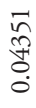 & 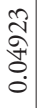 & 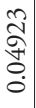 & 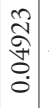 & 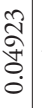 & 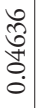 & 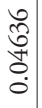 & 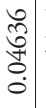 & 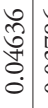 & $\begin{array}{l}0 \\
2 \\
\hat{n} \\
0 \\
0 \\
0\end{array}$ & 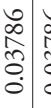 & $\begin{array}{c}0 \\
\infty \\
\vdots \\
c \\
\vdots \\
\vdots\end{array}$ & 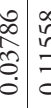 & 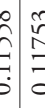 \\
\hline$N$ & & 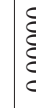 & 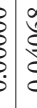 & 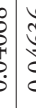 & $\begin{array}{l}0 \\
0 \\
0 \\
0 \\
0 \\
.\end{array}$ & \begin{tabular}{l}
0 \\
\multirow{2}{0}{} \\
$\vdots$ \\
0 \\
0
\end{tabular} & : & $\begin{array}{c}3 \\
0 \\
0 \\
0\end{array}$ & ঠ్ర్రా & $\sqrt{\tilde{f}}$ & $\begin{array}{l}\vec{\approx} \\
\stackrel{f}{J}\end{array}$ & 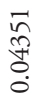 & $\begin{array}{l}\overline{\tilde{n}} \\
\hat{\sigma} \\
0 \\
0\end{array}$ & 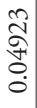 & 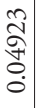 & 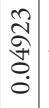 & 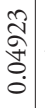 & 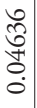 & $\begin{array}{l}\text { ô } \\
0 \\
0 \\
0 \\
0\end{array}$ & 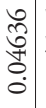 & 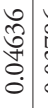 & $\begin{array}{l}0 \\
2 \\
0 \\
0 \\
0 \\
0\end{array}$ & 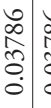 & 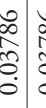 & 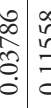 & 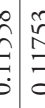 \\
\hline- & 8 & 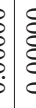 & 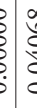 & 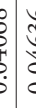 & $\begin{array}{l}0 \\
0 \\
0 \\
0 \\
0 \\
.\end{array}$ & $\begin{array}{c}0 \\
\widehat{6} \\
0 \\
0 \\
0\end{array}$ & $\begin{array}{l}0 \\
0 \\
0 \\
0\end{array}$ & $\begin{array}{l}0 \\
0 \\
0 \\
\vdots \\
0\end{array}$ & శ్రి & $\underset{\widetilde{n}}{\tilde{\sigma}}$ & $\underset{\widetilde{\Omega}}{\stackrel{\nabla}{f}}$ & 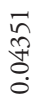 & 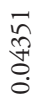 & 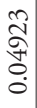 & 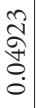 & 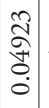 & 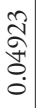 & 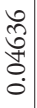 & 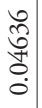 & 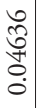 & 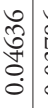 & $\begin{array}{l}0 \\
\infty \\
\hat{0} \\
0 \\
0\end{array}$ & 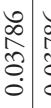 & 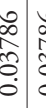 & 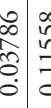 & 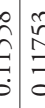 \\
\hline & 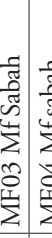 & 焉 & 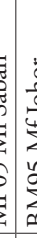 & 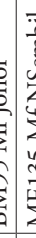 & 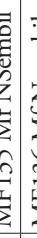 & 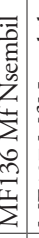 & 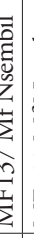 & \pm & $\frac{\pi}{\tilde{V}}$ & $\begin{array}{l}\vec{\Sigma} \\
\stackrel{\vec{\lambda}}{\lambda}\end{array}$ & 氶 & 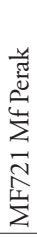 & 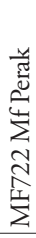 & 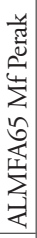 & 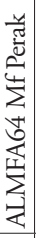 & 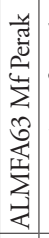 & 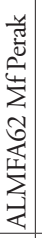 & 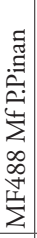 & 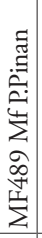 & 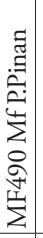 & 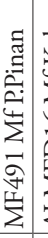 & 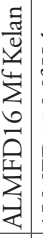 & 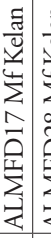 & 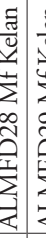 & 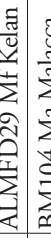 & 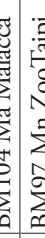 \\
\hline & & & & & & & & & & & & $\simeq$ & 2 & -1 & $\simeq$ & $\stackrel{2}{2}$ & 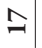 & $\stackrel{\infty}{=}$ & 2 & $\stackrel{\sim}{2}$ & $\overrightarrow{\mathrm{N}}$ & & $\tilde{\boldsymbol{\imath}}$ & $\underset{\sim}{*}$ & $\approx$ & ? \\
\hline
\end{tabular}




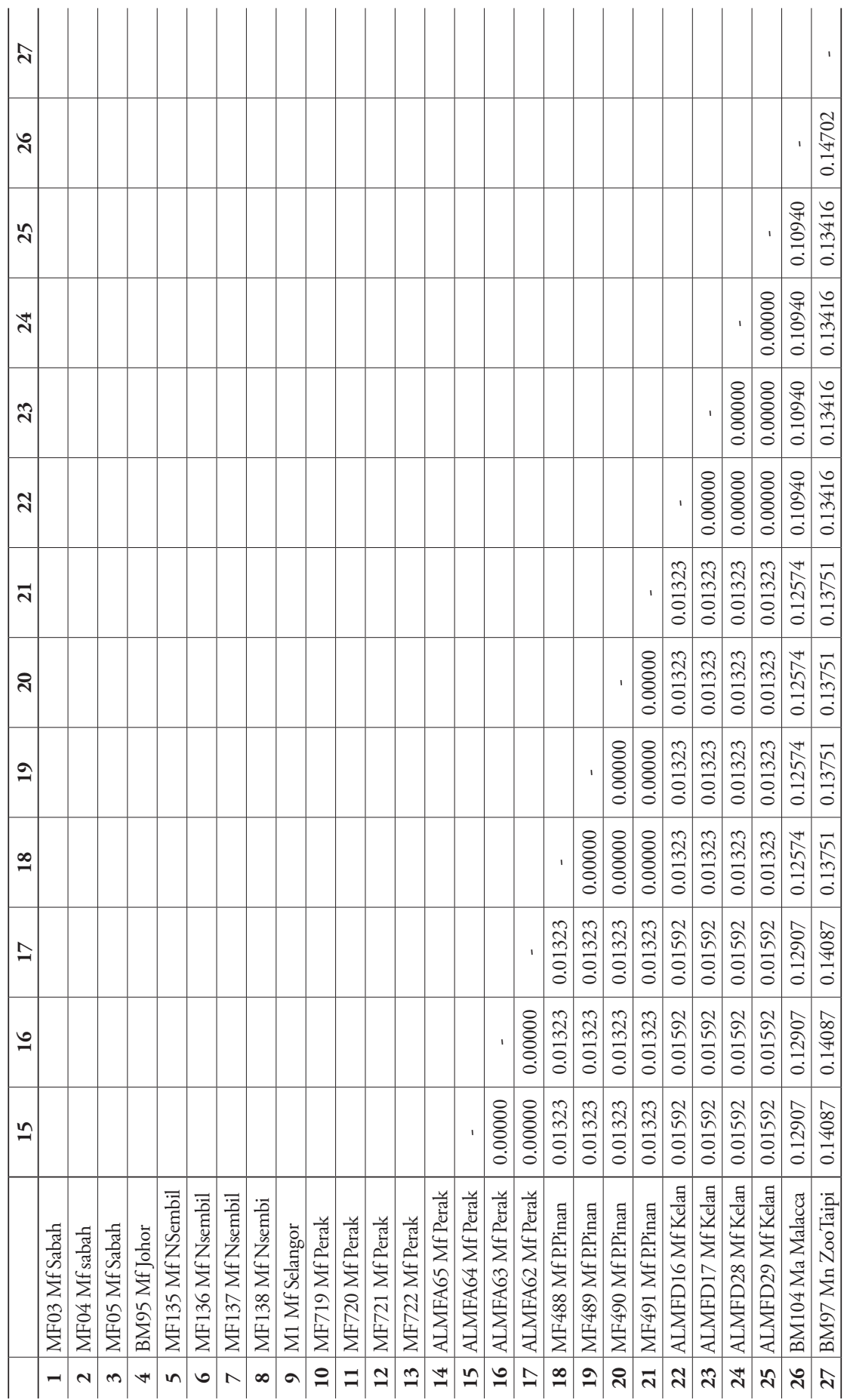


Table 3. Measures of nucleotide diversity $(\pi)$ and net nucleotide divergence among populations of $M$. fascicularis analyzed by locality.

\begin{tabular}{l|c|c}
\hline \multicolumn{1}{c|}{ Locality } & Nucleotide Diversity $(\boldsymbol{\pi})$ & Net nucleotide divergence (Da) \\
\hline Sabah-Johor & 0.01958 & 0.03916 \\
\hline Sabah-Negeri Sembilan & 0.02536 & 0.04439 \\
\hline Sabah-Selangor & 0.02219 & 0.04439 \\
\hline Sabah-Perak & 0.02089 & 0.04289 \\
\hline Sabah-Pulau Pinang & 0.02536 & 0.04439 \\
\hline Sabah-Kelantan & 0.02089 & 0.03655 \\
\hline Johor-Negeri Sembilan & 0.00209 & 0.00522 \\
\hline Johor-Selangor & 0.00522 & 0.00522 \\
\hline Johor-Perak & 0.00348 & 0.00373 \\
\hline Johor-Pulau Pinang & 0.00418 & 0.01044 \\
\hline Johor-Kelantan & 0.00313 & 0.00783 \\
\hline Negeri Sembilan-Selangor & 0.00000 & 0.00000 \\
\hline Negeri Sembilan-Perak & 0.00633 & 0.00895 \\
\hline Negeri Sembilan-Pulau Pinang & 0.00895 & 0.01567 \\
\hline Negeri Sembilan-Kelantan & 0.00746 & 0.01305 \\
\hline Selangor-Perak & 0.00464 & 0.00895 \\
\hline Selangor-Pulau Pinang & 0.00627 & 0.01567 \\
\hline Selangor-Kelantan & 0.00522 & 0.01305 \\
\hline Perak-Pulau Pinang & 0.00633 & 0.00895 \\
\hline Perak-Kelantan & 0.00760 & 0.01156 \\
\hline Pulau Pinang-Kelantan & 0.00746 & 0.01305 \\
\hline & &
\end{tabular}

Table 4. Measures of nucleotide diversity $(\pi)$ and net nucleotide divergence among populations of $M$. fascicularis analyzed by regions.

\begin{tabular}{l|c|c}
\hline \multicolumn{1}{c|}{ Regions } & Nucleotide Diversity $(\boldsymbol{\pi})$ & Net nucleotide divergence (Da) \\
\hline Peninsula Malaysia-Borneo & 0.01666 & 0.03801 \\
\hline East Coast-West Coast & 0.00943 & 0.00865 \\
\hline Mainland-Island & 0.00823 & 0.00918 \\
\hline
\end{tabular}

Nucleotide diversity $(\pi)$ and net nucleotide divergence $(\mathrm{Da})$ were also calculated for the Cyt $b$ sequence obtained using DnaSP v4.0. Two separate analyses for $\pi$ and Da were conducted based on the origin of samples, by first sorting the sequences (excluding outgroup) according to their locality (states) (Table 3) and then according to regions (Table 4). The first analysis (Table 3) portrayed that $\pi$ was the highest between Sabah and other states ranging from 0.019 to 0.025 , and the results are consistent with $\mathrm{Da}$, ranging from 0.037 to 0.044 . $\pi$. The lowest values, 0 for $\pi$ and $\mathrm{Da}$, were found between Negeri Sembilan and Selangor. The second analysis (Table 4) revealed that the Peninsula Malaysia and Borneo populations have $0.017 \pi$ and $0.038 \mathrm{Da}$, which are the highest compared to other regions; the East Coast of Peninsula Malaysia and West Coast of Peninsula Malaysia populations have $0.09 \pi$ and $0.08 \mathrm{Da}$ and mainland and island populations have $0.08 \pi$ and $0.09 \mathrm{Da}$, respectively. 


\section{Phylogenetic trees}

\section{Neighbor joining}

The NJ phylogeny tree (Figure 3) was generated using Kimura-2-Parameter with 1000 bootstrap replication. The NJ phylogenetic tree showed that samples originating from Borneo remain monophyletic from samples originating from Peninsula Malaysia, supported with $99 \%$ bootstrap value. Samples from Peninsula Malaysia were divided into 2 clades; clade A and Clade B. Clade A portrays the separation of samples originating from the East Coast of Peninsula Malaysia from the remaining samples with $64 \%$ bootstrap value. Clade $\mathrm{B}$ on the other hand is the assemblage of populations of the West Coast of Peninsula Malaysia, supported by $90 \%$ bootstrap value. Within Clade B, two further clades were defined, namely island population (Pulau Pinang) and mainland populations (Perak, Negeri Sembilan, Johor, and Selangor) supported by $93 \%$ and

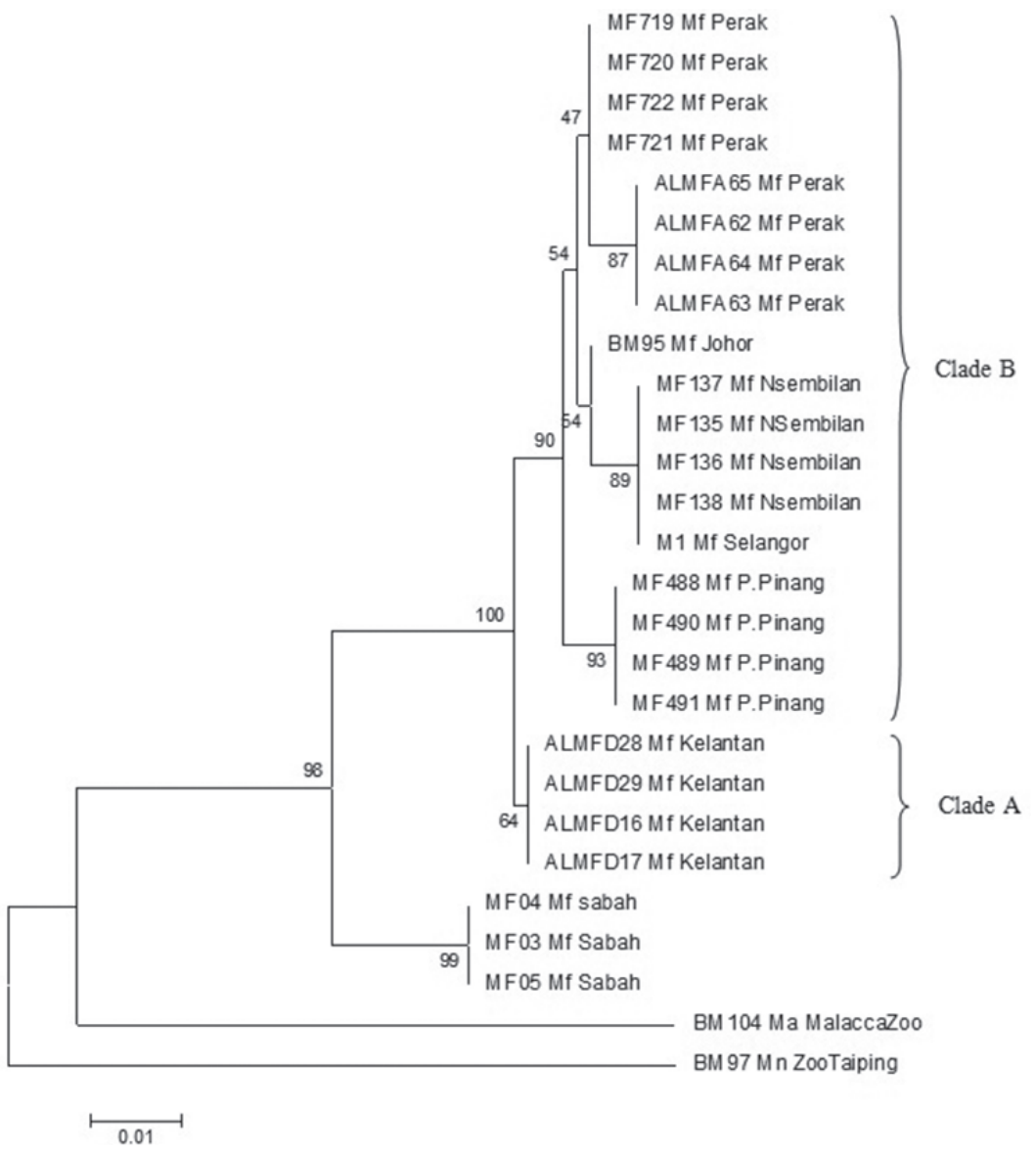

Figure 3. Neighbor joining phylogenetic tree using Kimura-2-Parameter algorithm with bootstrap values indicated on the branch. 


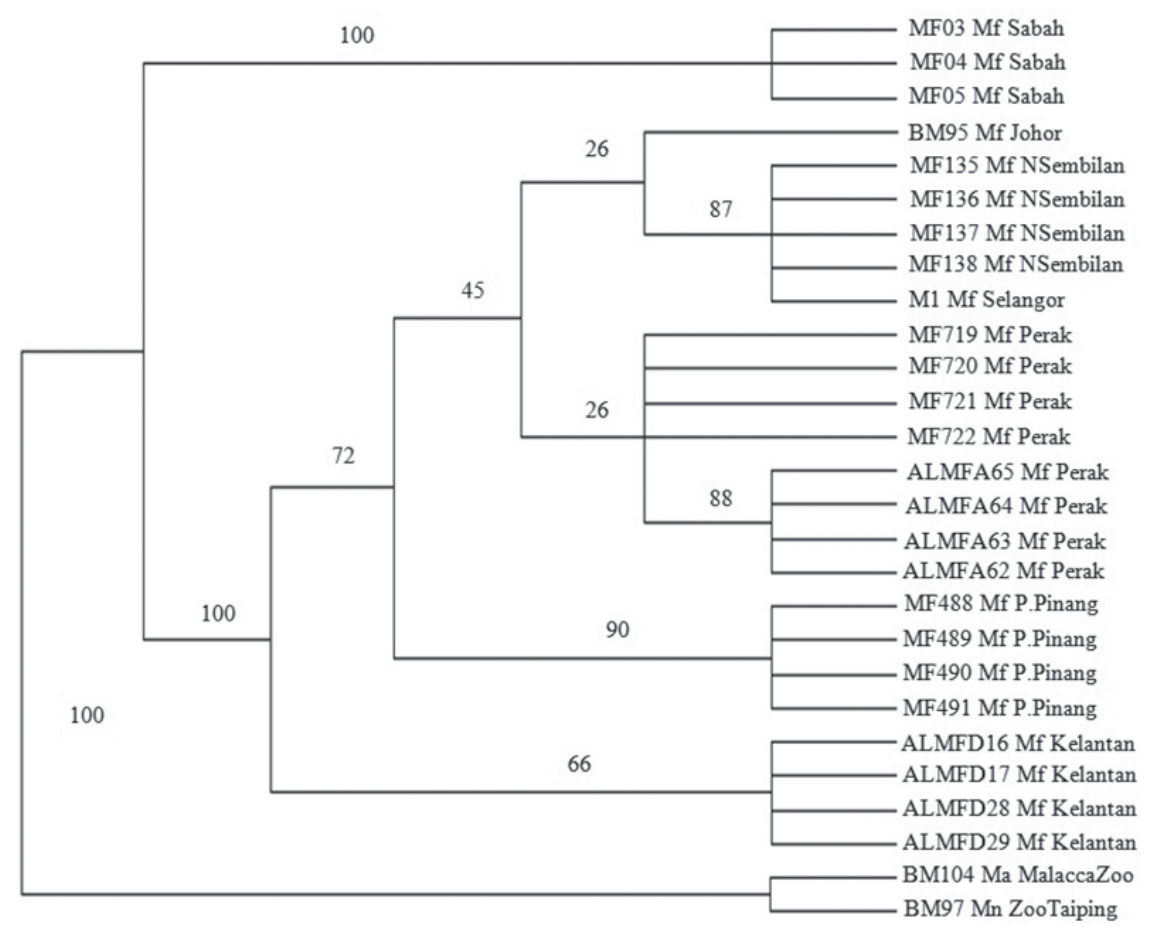

Figure 4. The Bootstrap 50\% majority rule consensus maximum parsimony tree of $M$. fascicularis populations. Bootstrap values are indicated on the branch.

54\% bootstrap value respectively. Populations from southern Peninsula (Negeri Sembilan, Johor and Selangor) and northern Peninsula (Perak) also were distinguished by $54 \%$ and $47 \%$ bootstrap value correspondingly.

\section{Maximum parsimony}

MP (Figure 4) analysis was conducted using PAUP $4.0(\mathrm{CI}=0.929, \mathrm{HI}=0.071$, RI $=0.944, \mathrm{RC}=0.878$ and tree length $=85) . M$. fascicularis populations were separated into 2 main clades, a Borneo clade and a Peninsula Malaysia clade both supported by $100 \%$ bootstrap value in the MP tree. Peninsula Malaysia's population was further divided into 2 sub clades, the East Coast of Peninsula Malaysia and West Coast of Peninsula Malaysia populations supported by $66 \%$ and $72 \%$ bootstrap value respectively. The West Coast of Peninsula Malaysia populations were further divided into 2 clades, comprising the mainland and island populations (Pulau Pinang) supported by $45 \%$ and $90 \%$ bootstrap value. The Northern Peninsula (Perak) and Southern Peninsula (Selangor, Negeri Sembilan and Johor) clades were also separated, both supported by $26 \%$ bootstrap value. 


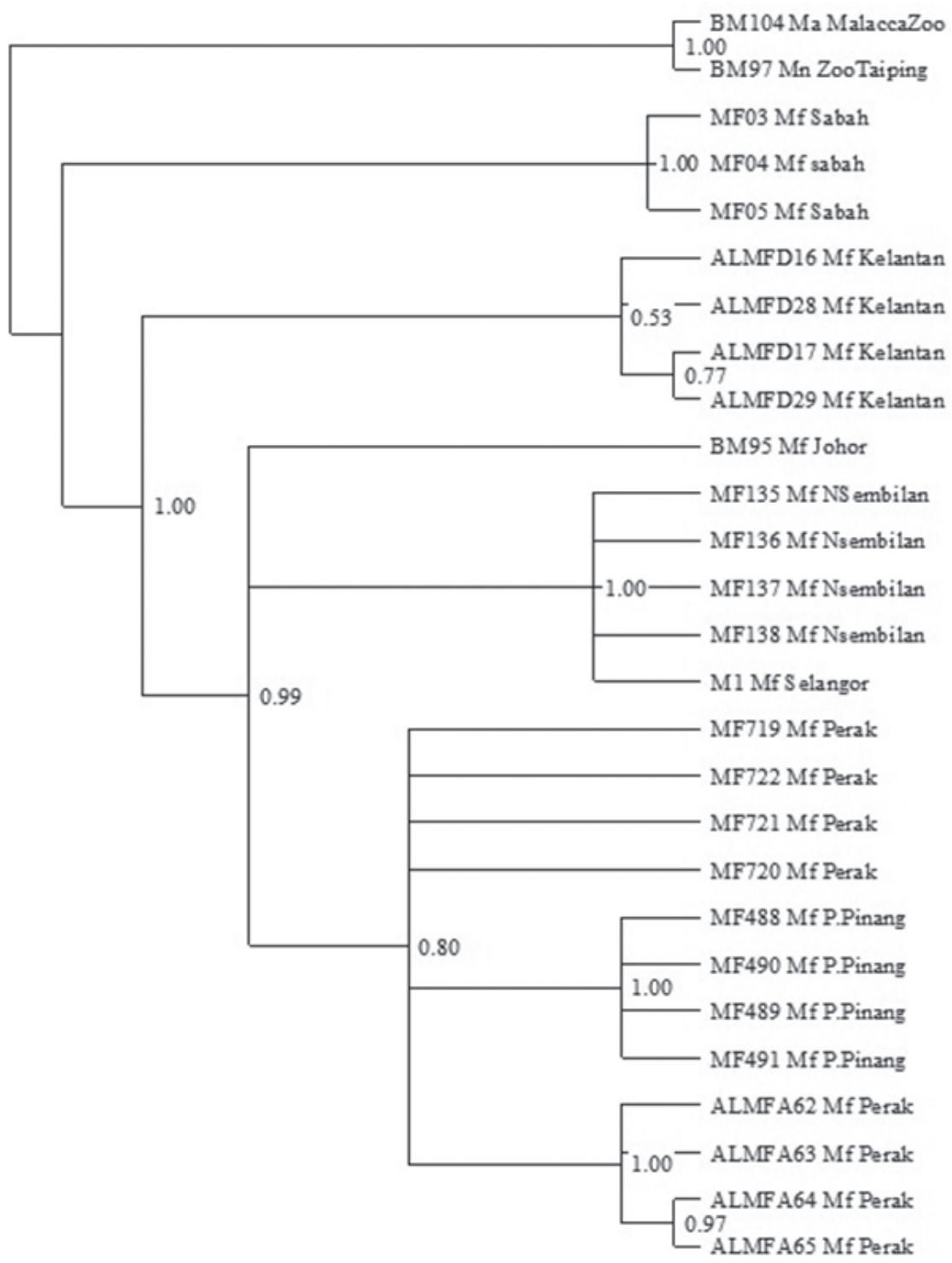

Figure 5. Bayesian inference of the $50 \%$ majority rule consensus tree of Cyt $b$ sequence of $M$. fascicularis populations with Bayesian posterior probability (PP) are accordingly indicated on the branch.

\section{Bayesian inference}

Bayesian inference phylogenetic tree (Figure 5) results are generally consistent with neighbor joining and maximum parsimony phylogenetic trees. Separations of M. fascicularis populations originating from Borneo and Peninsula Malaysia correspond well with other phylogenetic trees. Within M. fascicularis populations from Peninsula Malaysia, the East 
Coast of Peninsula Malaysia populations are yet again separated from the West Coast of Peninsula Malaysia populations with 0.53 and 0.99 posterior probabilities respectively. The island populations (Pulau Pinang), on the other hand, seem to group together with other mainland populations from the West Coast of Peninsula Malaysia. These are thoroughly inconsistent with both neighbor joining and maximum parsimony phylogenetic trees. The populations from Negeri Sembilan, Selangor and Johor are also distinguished from $M$. fascicularis populations from Perak and Pulau Pinang, supported by a 1.00 and 0.80 posterior probability.

\section{Discussion}

Sequence analysis of partial Cyt $b$ sequences indicates that this locus is highly conserved which yielded only 78 (20.37\%) variable sites among which only 34 (8.88\%) of parsimony informative characters across $M$. fascicularis, $M$. nemestrina and $M$. arctoides. Vun et al. (2011) reported 156 (40.21\%) variable sites and 124 (31.96\%) parsimony informative sites in the partial Cyt $b$ gene $(388 \mathrm{bp})$ across Presbytis and Trachypithecus (Colobinae). These findings are much higher compared to findings in this research, although analysis in this research was done within one genus while Vun et al. (2011) analyzed across 2 genera. Haus et al. (2013) analyzed $28.86 \%$ variable sites and $19.82 \%$ parsimony informative sites from full Cyt $b$ sequences in Chlorocebus, which are parallel with our findings, indicating Cyt $b$ is a highly conserved gene. Mitochondria are well known to play a central role in a variety of cellular processes and are the main source of adenosine triphosphate (ATP) (Kujoth et al. 2005). Cyt $b$ is a functional gene that contains both redox centers, $\mathrm{Q}_{0}$ and $\mathrm{Q}_{\mathrm{i}}$, involved in electron transfer (Hatefi 1985; Howell and Gilbert 1988). Thus, even a single point mutation that occurs in this region will probably have a deleterious effect on the functional response of the locus.

Macaca fascicularis populations in Malaysia are evidently divided into 2 main clusters: the Borneo populations and Peninsula Malaysia populations. Thirteen parsimony informative characters were detected between these populations from a total of 23 parsimony informative characters. Pairwise genetic distance analysis indicated that the genetic distance between these 2 populations was the highest as compared to other populations in Malaysia with a value of 0.041-0.049, aside from monophyletic states of both populations in all phylogenetic trees. These findings are highly anticipated, considering the vicariance theory on population disjunction as the central thesis. In this scenario, the Borneo populations and Peninsula Malaysia populations are separated by the South China Sea. This separation more than likely caused the interruption of gene flow between both populations, causing them to accrue the major genetic differences observed in this study.

Populations of $M$. fascicularis in Peninsula Malaysia also form 2 further subgroups: east and west. The population from Kelantan forms a single clade in all phylogenetic trees and genetic analysis in comparison to the rest of the Peninsula Malaysia populations. Thus, the Kelantan population likely represents a unique lineage as compared 
to other populations from Peninsula Malaysia. Results obtained by Vun et al. (2011) that used the same primers as in this research to study the phylogenetic relationships of genus Presbytis in Malaysia provide a suitable comparison. Vun et al. (2011) obtained a pairwise genetic distance (Kimura-2-Parameter) between Presbytis melalophos robinsoni and Presbytis melalophos siamensis as low as 0.058, and each of these subspecies has uniquely distinct morphological characteristics. In comparison, the pairwise genetic distance observed here between M. fascicularis populations of East and West coast of Peninsula Malaysia are 0.016; however, there are no documented morphological characteristics that differentiate these populations.

The observed genetic differences between populations of $M$. fascicularis are best viewed as subspecies separations. M. f. laeta (Elliot, 1909) was recorded by Medway (1969) as common on Tioman Island (the East Coast of Peninsula Malaysia), but a survey conducted by the Department of Wildlife and Nature Park, Malaysia, PERHILITAN (1995) proved otherwise. This particular subspecies was observed based on morphological characters on the mainland East Coast of Peninsula Malaysia by PERHILITAN (1995) as well as Weitzel et al. (1988), whose study also acknowledged the distribution of $M$. f. laeta in the East Coast of Peninsula Malaysia. Raven (1935), however, acknowledges $M$. f. argentimembris (Kloss, 1911) observed in Redang Island as the subspecies distributed in the East Coast of Peninsula Malaysia. Although these 2 classifications contradict each other on one dimension, both may be true. It may be possible that $M$. f. argentimembris are distributed in the North East Coast of Peninsula Malaysia and M. f. laeta are distributed in the South East Coast of Peninsula Malaysia. These are the most relevant classifications according to phylogenetic and genetic results of this study, which suggests that the Pasir Mas population is M. f. argentimembris. In contrast, the populations of Kluang, Pantai Remis, Kuala Gula, Port Dickson, and Bangi distributed on the west coast should be $M$. f. fascicularis.

The results of this study are directly relevant to conservation management strategies of M. fascicularis in Malaysia, particularly in terms of the translocation process. The human-macaque conflict has intensified in Malaysia due to habitat degradation and habituation of the species. It is important to recognize the genetic diversity between populations of $M$. fascicularis so we are able to conserve the unique evolutionary lineages of the species. Thus, PERHILITAN can translocate the target populations of $M$. fascicularis within the same gene pool of the other populations (the Borneo-Peninsula; east-west; mainland-island and north-south populations). This translocation is important to avoid the loss of genetic diversity that cannot be recovered, or, in extreme cases, can result in hybridization of populations and potential outbreeding depression of the populations (DeSalle and Amato 2004).

\section{Conclusion}

The phylogenetic relationships of Malaysia's long-tailed macaques, M. fascicularis, are crucial, as they are at a center of the human-wildlife crisis in the field of primatology, 
taxonomy and conservation biology. This research has shown that populations of $M$. fascicularis originating from Borneo and Peninsula Malaysia are distinguishable from each other based on genetic data. Within Peninsula Malaysia, further division occurs between the East Coast and West Coast of Peninsula Malaysia; mainland and island and Northern and Southern Peninsula populations. These data can aid the conservation management planning in terms of translocation of the target pest populations within similar gene pools. Further mtDNA and nuclear DNA studies of $M$. fascicularis at the population level are required to increase the number of individuals from each locality and increase the number of geographical locations to represent clades (east-west and Peninsula-Borneo), in which biogeographical factors can also be taken into account.

\section{Acknowledgment}

We thank to Zamzuriada AS and Zahar $\mathrm{N}$ for their laboratory contribution. We are deeply indebted to Department of Wildlife and National Parks that provided us with the necessary facilities and assistance for genetic samplings and permit to conduct this research with permit reference number (JPHL\&TN(IP): 80-4/2. We would like to thank the Sabah Parks and Sabah Biodiversity Centre. The authors acknowledge Universiti Kebangsaan Malaysia for providing necessary funding, facilities and assistance. This research was supported by grants FRGS/1/2012/STWN10/UKM/02/3, UKMGUP-2011-168, KOMUNITI-2011-023, ERGS/1/2013/STWN10/UKM/02/1 and DLP-2013-006.

\section{References}

Blancher A, Bonhomme M, Crouau-Roy B, Kitano T, Saitou N, Terao K (2008) Mitochondrial DNA sequence phylogeny of 4 populations of the widely distributed cynomolgus macaque (Macaca fascicularis fascicularis). Journal of Heredity 99(3): 254-264. doi: 10.1093/jhered/esn003

Brandon-Jones D, Eudey AA, Geissmann T, Groves CP, Melnick DJ, Morales JC, Shekelle M, Stewart CB (2004) Asian primate classification. International Journal of Primatology 25(1): 97-164. doi: 10.1023/B:IJOP.0000014647.18720.32

Crombie RI, Pregill GK (1999) A Checklist of the Herpetofauna of the Palau Islands (Republic of Belau), Oceania. Herpetological Monographs 13: 29-80. doi: 10.2307/1467060

Deinard A, Smith DG (2001) Phylogenetic relationships among the macaques: evidence from the nuclear locus NRAMP1. Journal of Human Evolution 41(1): 45-59. doi: 10.1006/ jhev.2001.0480

DeSalle R, Amato G (2004) The expansion of conservation genetics. Nature Reviews Genetic 5:702-712. doi: $10.1038 / \mathrm{nrg} 1425$

Fooden J (1996) Zoogeography of Vietnamese primates. International Journal of Primatology 17: 845-899. doi: 10.1007/BF02735268 
Groves CP (2001) Primate taxonomy. Smithsonian Institution Press, Washington, 350 pp.

Gumert MD, Fuentes A, Engel GA, Jones-Engel L (2011) Future direction for research and conservation of long-tailed macaque populations. In: Fuentes A, Gumert M, Jones-Engel L (Eds) Monkeys on the edge: ecology and management of long-tailed macaques and their interface with humans. Cambridge University Press, Cambridge, 328-353.

Hatefi Y (1985) The mitochondrial electron transport and oxidative phosphorylation system. Annual Review of Biochemistry 54: 1015-1069. doi: 10.1146/annurev.bi.54.070185.005055

Haus T, Akom E, Agwanda B, Hofreiter M, Roos C, Zinner D (2013) Mitochondrial diversity and distribution of African green monkeys (Chlorocebus Gray, 1870). American Journal of Primatology 75(4): 350-60. doi: 10.1002/ajp.22113

Howell N, Gilbert K (1988) Mutational analysis of the mouse mitochondrial Cytochrome $b$ and their possible roles in redox catalysis. Journal of Molecular Evolution 29: 157-169. doi: $10.1007 / \mathrm{BF} 02100114$

Huelsenbeck JP, Ronquist F (2001) MrBayes: Bayesian inference of phylogeny. Bioinformatics 17: 754-755. doi: 10.1093/bioinformatics/17.8.754

Kemp NJ, Burnett JB (2003) A biodiversity risk assessment and recommendations for risk management of Long-tailed Macaques (Macaca fascicularis) in New Guinea. December 2003. Indo-Pacific Conservation Alliance, Washington, DC, 116 pp.

Kocher TD, Thomas WK, Meyer A, Edwards SV, Pääbo S, Villablanca FX, Wilson AC (1989) Dynamics of mitochondrial DNA evolution in animal: Amplification and sequencing with conserved primers. Proceeding of the National Academy of Science of the United States of America 86: 6196-6200.

Kujoth GC, Hiona A, Pugh TD, Someya S, Panzer K, Wohlgemuth SE, Hofer T, Seo AY, Sullivan R, Jobling WA, Morrow JD, Remmen HV, Ssedivy JM, Yamasoba T, Tanokura M, Weindruch R, Leeuwenburgh C, Prolla TA (2005) Mitochondrial DNA mutations, oxidative stress, and apoptosis in mammalian aging. Science 309: 481-484. doi: 10.1126/ science. 1112125

Md-Zain BM, Mohamad M, Ernie-Muneerah MA, Ampeng A, Jasmi A, Lakim M, Mahani MC (2010a) Phylogenetic relationships of Malaysian monkeys, Cercopithecidae, based on mitochondrial cytochrome c sequences. Genetic and Molecular Research 9: 1987-1996. doi: 10.4238/vol9-4gmr942

Md-Zain BM, Sha ari NA, Mohd-Zaki M, Ruslin F, Idris NI, Kadderi MD, Idris WMR (2010b) A comprehensive population survey and daily activity budget on Long Tailed Macaques of Universiti Kebangsaan Malaysia. Journal of Biological Sciences 10(7): 608-615. doi: $10.3923 /$ jbs.2010.608.615

Md-Zain BM, Tarmizi MR, Mohd-Zaki M (2011) Campus monkeys of Universiti Kebangsaan Malaysia: Nuisance problems and student's perception. In: Fuentes A, Gumert M, Jones-Engel L (Eds) Monkeys on the edge: ecology and management of long-tailed macaques and their interface with humans. Cambridge University Press, Cambridge, 101117. doi: 10.1007/978-1-4614-3967-7_14

Medway L (1969) The wild mammals of Malaya and offshore islands, including Singapore. Oxford University Press, Kuala Lumpur. 
Otting N, Doxiadis GG, Ronald EB (2009) Definition of Mafa-A and-B haplotypes in pedigreed cynomolgus macaques (Macaca fascicularis). Immunogenetic 61(11-12): 745-753. doi: 10.1007/s00251-009-0412-9

Pääbo S, Wilson AC (1988) Polymerase chain reaction reveals cloning artifacts. Nature 334: 387-388. doi: 10.1038/334387b0

PERHILITAN (1995) Inventori kepelbagaian biologi Rezab hidupan liar Pulau Tioman. Biokepelbagaian mamalia di Pulau Tioman. Unpublished report 27-38.

PERHILITAN (2010) Annual Report, Department of Wildlife and National Parks (PERHILITAN) 38-39.

Posada D, Crandall KA (1998) MODELTEST: testing the model of DNA substitution. Bioinformatics 14: 817-818. doi: 10.1093/bioinformatics/14.9.817

Raven HC (1935) Wallace's Line and the Distribution of Indo-Australian Mammals. New York Press.

Rosli MKA, Syed-Shabthar SMF, Abdul-Patah P, Abdul-Samad Z, Abdul SN, Burhanuddin MN, Zulkifli NA, Shukor MN, Budsabong K, Changtragoon S, Sekiguchi T, Sasaki H, Md-Zain BM (2014) A New Subspecies Identification and Population Study of the Asian Small-Clawed Otter (Aonyx cinereus) in Malay Peninsula and Southern Thailand Based on Fecal DNA Method. The ScientificWorld Journal 2014: Article ID 457350. doi: $10.1155 / 2014 / 457350$

Rozas J, Sa'nchez-DelBarrio JC, Messeguer X, Rozas R (2003) DNAsp, DNA polymorphism analyses by the coalescent and other methods. Bioinformatics 19: 2496-2497. doi: 10.1093/bioinformatics/btg359

Smith DG, McDonough JW, George DA (2007) Mitochondrial DNA variation within and among regional populations of long tail macaques (Macaca fascicularis) in relation to other species of the fascicularis group of macaques. American Journal of Primatology 69(2): 182-198. doi: 10.1002/ajp.20337

Stevison LS, Kohn MH (2009) Divergence population genetic analysis of hybridization between rhesus and cynomolgus macaques. Molecular Ecology 18(11): 2457-2475. doi: 10.1111/j.1365-294X.2009.04212.x

Street SL, Kyes RC, Grant R, Ferguson B (2007) Single nucleotide polymorphisms (SNPs) are highly conserved in rhesus (Macaca mulatta) and cynomolgus (Macaca fascicularis) macaques. BMC genomics 8(1): 480. doi: 10.1186/1471-2164-8-480

Swofford DL (2002) Phylogenetic Analysis Using Parsimony (and Other Methods) Version 4.0 Beta. Sinauer Associates, Sunderland.

Tamura K, Dudley J, Nei M, Kumar S (2007) MEGA4: Molecular Evolutionary Genetics Analysis (MEGA) software version 4.0. Molecular Biology and Evolution 24: 1596-1599. doi: $10.1093 / \mathrm{molbev} / \mathrm{msm} 092$

Tosi AJ, Morales JC, Melnick DJ (2002) Y-chromosome and mitochondrial markers in Macaca fascicularis indicate introgression with $M$. mulatta and a biogeographic barrier in the Isthmus of Kra. International Journal of Primatology 23: 161-178. doi: 10.1023/A:1013258109954

Trask JS, George D, Houghton P, Kanthaswamy S, Smith DG (2013) Population and landscape genetics of an introduced species (M. fascicularis) on the island of Mauritius. PLoS ONE 8(1): 1-7. doi: 10.1371/journal.pone.0053001 
Vun VF, Mahani MC, Lakim M, Ampeng A, Md-Zain BM (2011) Phylogenetic relationships of leaf monkeys (Presbytis; Colobinae) based on cytochrome $b$ and 12S rRNA genes. Genetic and Molecular Research 10(1): 368-381. doi: 10.4238/vol10-1gmr1048

Weitzel V, Yang CM, Groves CP (1988) A catalogue of primates in the Singapore Zoological Reference Collection. The Raffles Bulletin of Zoology 36 (1): 1-166.

Zhang YP, Pan RL, Jablonski NG (1993) Classification and evolution of Asian Colobines. Folia Primatologica 60: 106-117. doi: 10.1159/000156680 